\title{
Effect of Arabic gum, xanthan gum and orange oil on flavor release from diluted orange beverage emulsion
}

\begin{abstract}
The influence of main emulsion components namely Arabic gum (13-20\% w/w), xanthan gum $(0.3-0.20 \% \mathrm{w} / \mathrm{w})$ and orange oil $(10-14 \% \mathrm{w} / \mathrm{w})$ on semi-quantitative headspace analysis of target volatile flavor compounds released from a model orange beverage (diluted orange beverage emulsion) was evaluated by using a three-factor circumscribed central composite design (CCCD). For optimization procedure, the peak area of 13 volatile flavor compounds (i.e. ethyl acetate, $\alpha$-pinene, ethyl butyrate, $\beta$-pinene, 3-carene, myrcene, limonene, $\gamma$ terpinene, octanal, decanal, linalool, neral and geranial) were considered as response variables. The response surface analysis exhibited that the significant $(p<0.05)$ second-order polynomial regression equations were successfully fitted for all response variables except for ethyl butyrate. A satisfactory coefficient of determination (R2) ranged from 0.831 to 0.969 $(>0.8)$ was obtained for the response variables studied. No significant $(\mathrm{p}>0.05)$ lack of fit was indicated for the reduced models except for the models fitted for limonene and linalool. This observation confirmed an accurate fitness of the reduced response surface models to the experimental data. The multiple response optimizations indicated that an orange beverage emulsion containing $15.87 \%(\mathrm{w} / \mathrm{w})$ Arabic gum, $0.5 \%(\mathrm{w} / \mathrm{w})$ xanthan gum and $10 \%(\mathrm{w} / \mathrm{w})$ orange oil was predicted to provide the minimum overall flavor release.
\end{abstract}

Keyword: Semi-quantitative analysis, Orange beverage emulsion, Central composite design, Orange oil, Response surface model, Flavor release 